\title{
A Simulation Study on the Focal Plane Detector of the LAUE Project
}

\author{
M. Kalil, F. Frontera, E. Caroli, \\ E. Virgilli, V. Valsan
}

Received: date / Accepted: date

\begin{abstract}
The LAUE project, supported by the Italian Space Agency (ASI), is devoteded to the development of a long focal length (even 20 meters or longer) Laue lens for gamma ray astronomy between $80-600 \mathrm{keV}$. These lenses take advantage of Bragg diffraction to focus radiation onto a small spot drastically improving the signal to noise and contemporary significantly reducing the required size of the detector. In this paper we present a Monte-Carlo simulation study with MEGALIB to optimize, for space applications, the detector size to achieve high detection efficiency, and to optimize the position resolution of the detector to reconstruct the Point Spread Function of the lens considered for the LAUE project. Then, using SILVACO semiconductor simulation toolkit, response simulations were made on the optimized detector to estimate its capacitance per channel and depletion voltage. In all of the simulations, two materials were compared; a low density material (Silicon) and a high density material (Germanium).
\end{abstract}

Keywords Point Spread Function · Laue - MEGALIB - Semiconductors · $\mathrm{X}$-rays $\cdot$ gamma-rays.

E. Virgilli · F. Frontera · P. Rosati

Department of Physics, University of Ferrara, Via Saragat 1/c, 44122 Ferrara, Italy E-mail: virgilli@fe.infn.it

E. Bonnini · E. Buffagni · C. Ferrari

CNR-IMEM Institute, Parco Area delle Scienze 37/A, 43124 Parma, Italy

N. Auricchio · E. Caroli · J. Stephen

INAF/IASF via Gobetti Bologna, Italy 


\section{INTRODUCTION}

Astrophysical observations above $80 \mathrm{keV}$ have been only performed by directviewing instruments whose sensitivity is limited by the background. Many astrophysical issues are still open at these energies [1]. For example, the origin of matter-antimatter annihilation emission at $511 \mathrm{keV}$ from the Galactic Center and the accrection physics in Active Galactic Nuclei above $100 \mathrm{keV}$. To increase the sensitivity, a new generation of broad band X-ray focusing telescopes that extend the operative energy up to several hundreds of $\mathrm{keV}$ is needed. The most efficient technique to focus photons above $80 \mathrm{keV}$ appears to be the Bragg diffraction from bent crystals in a Laue geometry. In general, when photons of energy E impinge on crystals with grazing angle with respect to a set of atomic planes of Miller index (hkl), they are diffracted according to the Bragg diffraction law:

\section{xxxxx}

he LAUE project [2], supported by the Italian Space Agency (ASI), is a project dedicated to create a technology to construct a Laue lens with a focal length of 20 meters able to focus photons in the 80-600 keV energy band. LAUE will be able to perform deep sensitive studies of astrophysical sources. It will, for example, disentangle the source physics, the emission mechanisms at work and discover new physics [3]. A Laue lens focuses gamma rays by using Bragg diffraction in the Laue geometry, i.e. in transmission. A large amount of crystals pieces are arranged in concentric rings and oriented to diffract the Xray radiation coming from a source at infinity toward a common point (Figure 1). Each crystal ring is symmetrical with respect to the line of sight of the lens. Perfect single crystals are not convenient for the construction of a Laue lens given that their response covers a very narrow energy band (corresponding to the Darwin width of the crystal). Mosaic flat crystals were the first attempt to overcome the pass band requirements. In mosaic crystals, thanks to the microcrystals orientation distribution with respect to a main direction, an energy pass band corresponding to 30 " 60 " is typically obtained. Bent crystals, instead, are the ideal elements to cover the entire lens surface, by obtaining a continuous (free of gaps) energy pass band.

The lens of our project has a spherical shape with radius $\mathrm{R}$ and focal length $\mathrm{f}=\mathrm{R} / 2$, and makes use of bent crystals with curvature radius equal to $\mathrm{R}$. In this configuration the lens becomes a real imaging focusing instrument, satisfying the Abbe law, as will be discussed elsewhere. In the lens focus a detector is positioned, hereafter called a focal plane detector. The focal plane detector, we assume for space astronomical observations thus a space worthy instrument, is formed of several layers of double sided strip detectors (DSSDs [5]). Because they are position sensitive, these detectors are able to reconstruct the point spread function (PSF) of a Laue lens, which describes the two dimensional distribution of photons in the focal plane of the lens for a point source at infinity. Using MEGALIB [6], we have performed a simulation on the focal plane detector, assumed to be $3 \mathrm{D}$ position sensitive. In this study two types 


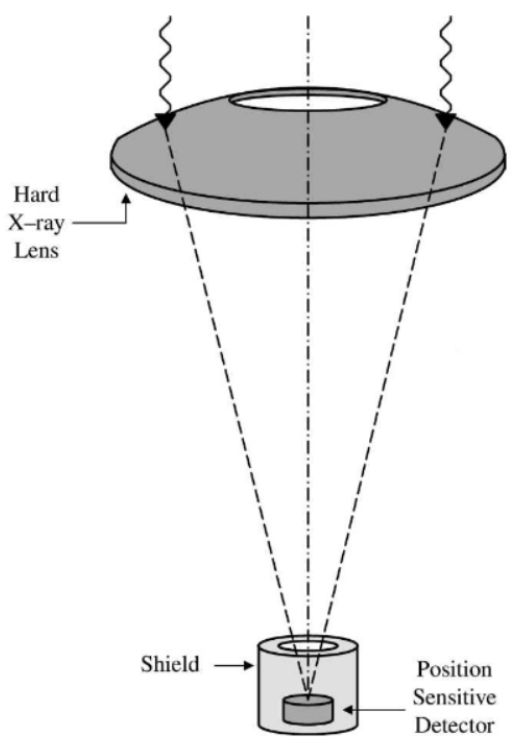

Fig. 1: Pictorial view of a Laue lens (not to scale). The lens is made of mosaic crystal tiles in transmission configuration. The impinging photons are diffracted by the crystal tiles and focused onto a small region centered in the focus of the lens where a position sensitive detector is positioned [4].

of material were compared: a high density material (Germanium) and a low density material (Silicon). The objectives of the simulation were:

- Optimizing the volume of the focal plane detector to achieve a detection efficiency of at least $80 \%$ for the $558-645 \mathrm{keV}$ photons (i.e. the photons at the upper threshold of the Lenss pass band).

- Optimizing the 2D position resolution in the focal plane in order to reconstruct the PSF under the condition that the peak intensity profile of the PSF is spread over at least 3 strips.

Hence the main goal of this paper is to optimize the coupling of the Laue lens and the detector in terms of efficiency and 2D spatial resolution. The depth resolution of the detector is assumed to be perfect and is not optimized in this work. In addition, the detector background will be studied and taken into account in future development of the simulation work that will be presented in this paper. Despite this, we will make some considerations on the expected background for each of the considered detector material. After optimizing the performance of the DSSDs with MEGALIB, the volume and pitch of the detectors were defined. Using the SILVACO [7] semiconductor simulation toolkit, the response of the optimized DSSDs was simulated to obtain the following information: 
- The required full depletion voltage.

- The noise of the detector: capacitance and leakage current.

In the following we present the results of this study.

\section{References}

